\title{
LA MOVILIDAD EN EL CAMPUS VILA-SECA DE LA URV: PROPUESTAS PARA UNA MOVILIDAD MÁS SOSTENIBLE
}

\author{
Òscar Saladié \\ Departamento de Geografía. Universitat Rovira i Virgili \\ Cátedra Dow/URV de Desarrollo Sostenible \\ oscar.saladie@urv.cat \\ Joan Jurado \\ Cátedra Dow/URV de Desarrollo Sostenible \\ joanjuradorota@gmail.com
}

\section{RESUMEN}

Mediante una encuesta a 191 estudiantes de los grados de Turismo y de Geografía y Ordenación del Territorio de la URV se ha analizado la movilidad durante el curso académico desde su lugar de residencia hasta el Campus Vila-seca. En un 64,4\% el desplazamiento se realiza en coche y el 67,5\% van solos en el vehículo. A mucha distancia del coche se encuentra el autobús (19,9\%). Un 7,3\% se desplazan en tren y un $6,8 \%$ a pie. Existen diferencias significativas teniendo en cuenta el municipio de residencia de los estudiantes. El principal motivo para escoger el coche es la rapidez en el desplazamiento (41,5\%), seguido de la poca oferta de transporte público (26\%). Por su parte, las principales razones esgrimidas entre los usuarios de autobús y tren son el precio (32,1\%) y el hecho de no disponer de vehículo propio (28,3\%). Seis de cada diez de los estudiantes que se desplazan en coche cambiarían a un medio de transporte colectivo si las razones aducidas para no hacerlo no se dieran. Finalmente, se proponen un total de seis acciones para alcanzar una movilidad más sostenible.

Palabras clave: Movilidad, sostenibilidad, estudiantes universitarios, Campus Vila-seca, Universitat Rovira i Virgili, URV.

\section{ABASTRACT}

\section{Mobility in Vila-seca Campus at URV: proposals towards a more sustainable mobility}

Student mobility in Vila-Seca Campus at Rovira i Virigili University has been analyzed by means of a survey conducted to 191 students of Tourism and Geography degrees. Almost two third of them indicated the trip from their place of residence during the academic year is made by car and the most of them travel alone. Only $19.9 \%$ take the bus, $7.3 \%$ the train and $6,8 \%$ walking. There are significant differences taking into account location of the students. The main reason for choosing the car is the speed of displacement (41.5\%), followed by the short supply of public transport (26\%). Meanwhile, the main reasons given among users of bus and train are the price $(32.1 \%)$ and the fact of not having own vehicle (28.3\%). Six out of ten who travel by car would change to public transport if the reasons given for not doing would not exists. Finally, a total of six actions are proposed to achieve a more sustainable mobility. URV.

Keywords: Mobility, sustainability, university students, Campus Vila-seca, University Rovira i Virgili,

\section{INTRODUCCIÓN}

Una de las características de la sociedad contemporánea es la gran movilidad de personas y mercancías. Esta cada vez mayor movilidad se ha producido en paralelo a la introducción de mejoras tanto en 
los vehículos como en las redes de transporte y comunicación. Un sector, el del transporte, que consume una gran parte de la energía final utilizada y que genera una gran cantidad de contaminantes, entre ellos el dióxido de carbono, principal gas de efecto invernadero causante del cambio climático (Chapman, 2007). A su vez, el transporte es una de las principales fuentes de contaminación acústica. Por todo ello, no es de extrañar que las variables ambientales hayan sido incluidas en las cuestiones relacionadas con la movilidad (Miralles-Guasch y Cebollada, 2009). Esta inclusión tiene por objetivo que la movilidad y el transporte sean más sostenibles, tal y como manifiestan, entre otros, Button y Nijkamp (1997), Roth y Kaberger (2002), Richardson (2005) y Banister (2008).

Greene y Wegener (1997) consideran que la planificación territorial es una de las piezas clave para alcanzar una movilidad más sostenible. Es decir, una vez analizada la evolución y la situación actual de un territorio hay que definir una serie de propuestas que permitan, con su aplicación, modificar y mejorar la realidad. El modelo de ocupación del territorio y, tal y como indica Banister (2008), la mayor descentralización de las ciudades, han propiciado un aumento en la movilidad y una elevada dependencia del coche. El mismo autor manifiesta que las ciudades deben diseñarse de manera que posibiliten una buena accesibilidad y una buena calidad ambiental. De acuerdo con Hickman, Hall y Banister (2013) las políticas de movilidad deben de estar integradas en los procesos de planificación, también urbana y de esta manera, tal y como concluyen Haque, Chin y Debnath (2013), será más fácil implementar un sistema de transporte más inteligente, más seguro y más sostenible. En definitiva, las variables relacionadas con el medio ambiente y la sostenibilidad plantean nuevos retos en la planificación de la movilidad, tal y como señalan Cebollada y Miralles-Guasch (2010), en el caso de la Región Metropolitana de Barcelona.

La mejora del transporte público es la acción clave para reducir la movilidad en coche tal y como afirman, entre otros, Hensher (1998) o Mackett (2001). Su deficiencia ha sido debida, en muchos casos, al modelo de crecimiento urbano (Camagni, Gibelli y Rigamonti, 2002; Catalán, Saurí y Serra, 2008; García-Palomares, 2010). La promoción del transporte público debe ir acompañada por la potenciación del uso compartido de los vehículos, tal y como manifiestan Efthymiou, Antoniou y Waddell (2013), especialmente por lo que respecta la movilidad diaria. Unas estrategias aún más necesarias debido a la perspectiva de un aumento del precio de los combustibles y a las dificultades de aparcamiento en los núcleos urbanos. No obstante, en muchas ocasiones no existen alternativas que permitan dejar de utilizar el coche (Mackett, 2003) y sustituirlo por transporte público colectivo o bicicleta. Una manera esta última que, junto a los desplazamientos a pie, son más plausibles en el caso de los desplazamientos urbanos y que junto a los beneficios ambientales también incorporan beneficios para la salud (Woodcock, Baniester, Edwards, Prentice y Robert, 2007; Villanueva, Giles-Corti y McCormack, 2008). Ahora bien, además de contar con un servicio eficiente de alquiler de bicicletas, es necesario que la movilidad por la ciudad en medios de transporte no motorizados sea segura (Pucher y Dijkstra, 2003; Pooley et al., 2013).

En el éxito de las políticas tendentes a conseguir una movilidad más sostenible y que propicien una mejora de la calidad de vida de los ciudadanos también juegan un papel muy importante las actitudes (Steg y Gifford, 2005). Unas actitudes que, en parte, determinan el comportamiento por lo que respecta a la movilidad y que muchas veces responde a factores y situaciones de carácter personal (Prillwitz y Barr, 2011). No obstante, tal y como indica Anable (2005), un mismo comportamiento puede ser atribuido a un amplio abanico de razones y, a su vez, unas mismas actitudes pueden conducir a comportamientos diferenciados. Eriksson, Garvill y Nordlund (2008) consideran que se reduciría más fácilmente el uso del coche si los conductores tuvieran una importante motivación moral para hacerlo, mientras que Ibeas, dell'Olio y Barreda (2011) indican que en la consecución de una movilidad más sostenible es clave la implicación directa de los ciudadanos en los procesos de planificación. Por su parte, Köhler et al. (2009) argumentan que es más probable que la consecución de una movilidad más sostenible sea debida a cambios tecnológicos que a cambios en el estilo de vida de los ciudadanos. Conclusión que no comparten Moriarty y Honnery (2008) que consideran que las mejoras técnicas y tecnológicas en los vehículos (mayor eficiencia en el consumo de los derivados del petróleo o proliferación de modelos híbridos y eléctricos) no solucionarán los problemas de carácter ambiental debido al aumento continuado del parque automovilístico. Por su parte Vergragt y Szejnwald (2007) manifiestan que para alcanzar el objetivo de una movilidad más sostenible son necesarios tanto la innovación tecnológica como los cambios sociales y culturales.

Las universidades, tanto en el ámbito de la docencia como de la investigación y la gobernanza, juegan un papel clave en el fomento del desarrollo sostenible, ya sea por lo que respecta al propio funcionamiento de la universidad (Ferrer-Balas, 2004; Ferrer-Balas, Bruno, de Mingo y Sans, 2004; Velazquez, Munguia, Platt y Taddei, 2006; Evangelinos, Jones y Panoriou, 2009; Lukman, Tiwary y Azapagic, 2009; Emanuel y Adams, 2011; Lambrechts, Mulà, Ceulemans, Molderez y Gaeremynck, 2013; Lozano, Lukman, Lozano, 
Huisingh y Lambrechts, 2013; Nejati y Nejati, 2013) como también en relación al territorio del cual son referencia (Sedlacek, 2013). Los estudiantes, independientemente de en qué se han formado y a qué se dedicarán profesionalmente, deben ser conscientes que sus acciones y actitudes, ya sea como ciudadanos o como trabajadores, pueden tener afectación en el medio ambiente (Saladié, Alberich, Felipe y Aguilar 2013).

La utilización del vehículo privado es muy habitual en la movilidad de los estudiantes, los profesores y el personal de administración y servicios de los centros de educación superior en sus desplazamientos cotidianos hacia la universidad (Kerr, Lennon y Watson, 2010; Hancock y Nuttman, 2014). No obstante, su peso es desigual. En el caso de España encontramos valores tan dispares como, por ejemplo, un 27,1\% en el campus de Valladolid de la Universidad de Castilla y León (de las Rivas, Iglesias y Lalana, 2011), un 36,9\% en la Universitat Autònoma de Barcelona (Miralles-Guasch, Martínez y Marquet, 2012) o un 64,3\% en la Universitat de les Illes Balears (Seguí-Pons, Ruiz y Luna, 2013). Así pues, los mismos problemas ambientales indicados anteriormente pueden ser extrapolables y no es de extrañar que los órganos de dirección de los centros de educación superior desarrollen e implementen medidas tendentes a reducir la movilidad en coche de los miembros de la comunidad universitaria en el marco de planes más amplios tendentes a hacer más sostenibles las universidades en su conjunto.

Entre las razones por las cuales los estudiantes universitarios eligen el coche privado como medio de transporte para desplazarse se encuentra su rapidez y flexibilidad, contra las cuales no puede competir, en el caso de la Universidad de La Laguna, por ejemplo, el autobús (Alonso, 2002). La comodidad de no depender de un horario concreto para tomar el autobús o el tren también juega a favor del uso del coche. No obstante, en el caso del tren se evitan los posibles atascos de entrada y salida en horas punta. Pero a pesar de esto es necesario que haya una buena frecuencia, que la distancia a recorrer entre la estación de tren y el centro universitario no sea excesiva y que se minimicen los retrasos debido a averías o a otras causas.

El tiempo empleado para realizar el desplazamiento en transporte público es también un factor limitante según Miralles-Guasch y Domen (2010) entre los estudiantes de la Universitat Autònoma de Barcelona. Un tiempo que puede aumentar por la necesidad de realizar transbordos. En el caso del profesorado y el personal de administración y servicios de esta misma universidad catalana, Miralles-Guasch (2012) sostiene que el uso del coche no es tanto por las desventajas del transporte público, sino por la comodidad del usuario, a la que hay que añadir la percepción de cierto estatus por el hecho de usarlo y el placer asociado a la experiencia de dicho uso. Whalen, Páez y Carrasco (2013) consideran que, en el caso de los estudiantes de la McMaster University, la elección del modo de transporte es una combinación de costes, actitudes individuales y factores ambientales.

Tolley (1996), después de afirmar que los desplazamientos en coche tendrían que reducirse drásticamente, apunta como un hándicap el hecho que muchos campus universitarios disponen de plazas de aparcamiento gratuitas o a precios muy bajos. De esta manera, aumentar el precio del aparcamiento ha sido una estrategia identificada por la University of Idaho para desincentivar el uso del coche (Delmelle y Delmelle, 2012). Adaptar los precios y restringir el aparcamiento también se ha aplicado en la Universidad de Florida, aunque en paralelo han realizado una inversión importante en el transporte público que ha permitido aumentar su uso en casi un 300\% entre 1995 y 2003 (Bond y Steiner, 2006). Una combinación lógica, puesto que únicamente una restricción en el aparcamiento afectaría principalmente a los estudiantes con un menor poder adquisitivo y a aquellos que residen en localidades con una mala conectividad en transporte público entre su lugar de residencia y el centro universitario.

Bilbao y Fernández (2004) consideran que en el caso de la Universidad del País Vasco, concretamente en los centros situados en Bilbao, se produciría un aumento de nuevos usuarios del transporte público si hubiera una mayor frecuencia de tren y metro y si se redujera el precio en el caso del autobús. Un aumento que sería mayor si al lado del centro universitario hubiera una estación de trenes o de autobuses. Una cuestión clave esta última que, según Atherton y Giurco (2011), explica que únicamente un 7\% de los estudiantes de la University of Technology of Sidney utilizan el coche en sus desplazamientos mientras que más de la mitad escogen el tren. Por su parte, Kerr et al. (2010) indican que la principal estrategia a implementar tendría que incidir en el comportamiento de los usuarios del coche, especialmente en la percepción social que tiene su uso. Una conclusión en sintonía con la expresada por Miralles-Guasch et al. (2012) en el caso de la Universitat Autònoma de Barcelona, quienes consideran que dará un mejor resultado el cambio de las creencias y los valores sobre el coche, junto a la penalización de su uso, que las inversiones para que el transporte público sea más competitivo.

Los centros universitarios deberían tener entre sus objetivos conseguir una movilidad más sostenible y definir e implementar unas acciones que permitieran llevar a cabo dichos objetivos. Balsas (2003) ana- 
liza los resultados obtenidos en ocho campus universitarios de Estados Unidos de América tras la aplicación de sus respectivos planes. Pero desde la academia no únicamente se llevan a cabo este tipo de análisis, sino que también se realizan propuestas. Algunos ejemplos son las de Shannon et al. (2006) para la University of Western Australia, Pitsiava-Latinopoulou, Basbas y Gavanas (2013) para la Aristotle University of Thessaloniki o Atherton y Giurco (2011) para la University of Technology of Sidney. En este último caso los objetivos y las acciones propuestas no se circunscriben únicamente al transporte, sino también a la reducción de la utilización de papel y en hacer frente al cambio climático. Entre los objetivos en materia de transporte destacan evitar el desplazamiento cuando sea posible, aumentar el uso de los modos de transporte sostenibles y disminuir el uso del coche privado y disminuir la contaminación, especialmente las emisiones de gases de efecto invernadero. Mientras que entre las acciones se encuentran, aumentar y promover las videoconferencias y otras tecnologías para reducir la necesidad de viajar, promover políticas que permitan una mayor flexibilidad laboral, facilitar la movilidad en bicicleta (estacionamientos, duchas y taquillas), promover el uso de los medios de transporte públicos disponibles, incorporar descuentos en los bonos de los billetes del transporte público y fomentar el uso compartido del coche.

Para que las medidas planteadas anteriormente tengan éxito los usuarios han de estar informados sobre las diferentes opciones disponibles que les permitan reducir el uso del coche privado en sus desplazamientos. No obstante, según Brög, Erl y Mense (2004) es necesaria una aproximación más proactiva y que los organismos encargados de conseguir una movilidad más sostenible incidan (ayuda o consejo) en la toma de decisión del usuario para que escoja la opción más adecuada.

La Universitat Rovira i Virgili (URV) no es ajena al reto de conseguir una movilidad más sostenible entre sus miembros. El Plan de Medio Ambiente de la URV vio la luz a finales del año 2010. Su finalidad es "abordar la gestión ambiental desde un punto de vista integral y ambicioso, y explicitar su compromiso con el desarrollo sostenible" (ERF, 2010, p. 101) y su objetivo principal es reducir, en el horizonte del año 2020 , en un 20\% las emisiones de gases de efecto invernadero en relación a las del año 2005. Para alcanzar este objetivo se ha establecido un plan de acción con cinco líneas estratégicas (ERF, 2012): ahorro y eficiencia energética (LE1), mejora de la movilidad (LE2), comunicación del Plan de Medio Ambiente (LE3), ambientalización de la organización (LE4) y ambientalización curricular (LE5).

En el año 2012 el 68\% de las emisiones de gases de efecto invernadero en la URV correspondían a la movilidad, mientras que un $26 \%$ al consumo eléctrico y el restante $6 \%$ a otros conceptos (URV, 2013). La línea estratégica 2 del Plan de Medio Ambiente tiene por objetivo reducir un 10\% la cuota modal correspondiente al vehículo privado, para lo cual es necesario implantar el Plan de Movilidad de la URV que tiene por objetivos potenciar tanto el uso del transporte colectivo como los desplazamientos a pie y en bicicleta, fomentar el uso compartido del vehículo privado, alcanzar la accesibilidad universal en todos los centros universitarios e informar y sensibilizar a la población universitaria sobre las repercusiones ambientales asociadas a la movilidad.

La URV cuenta con un total de seis campus situados en diferentes municipios de la provincia de Tarragona. Dos de ellos se encuentran en Tarragona (Campus Catalunya y Campus Sescelades), dos en Reus (Campus Bellissens y Campus Vapor Vell), otro en Tortosa (Campus Terres de l'Ebre) y el sexto y último en Vila-seca (Campus Vila-seca). El Campus Vila-seca entró en funcionamiento durante el curso académico 2004-2005 con la Escuela Universitaria de Turismo y Ocio donde se impartía la diplomatura en Turismo. Posteriormente, a la Escuela Universitaria se le unió el Parque Científico y Tecnológico de Turismo y Ocio. Desde el curso académico 2012-2013 la Escuela Universitaria se ha convertido en la Facultad de Turismo y Geografía. Actualmente se imparten el grado en Turismo, el grado en Geografía y Ordenación del Territorio, el máster en Técnicas de Análisis e Innovación Turística y el máster en Planificación Territorial.

Tomando como punto de partida la información anterior, los objetivos de este estudio son, en primer lugar, analizar la movilidad de los estudiantes del Campus Vila-seca de la URV y, en segundo lugar, establecer una serie de propuestas para reducir el uso del automóvil privado y potenciar el uso de los medios de transporte colectivos y los desplazamientos a pie o en bicicleta. Para alcanzar los objetivos planteados el artículo se ha estructurado en cinco apartados principales, a los cuales siguen las conclusiones, los agradecimientos y la bibliografía. Una vez contextualizado el tema y planteados los objetivos en este primer apartado introductorio, en el segundo apartado se lleva a cabo un descripción de la localización de Vila-seca y del centro universitario de la URV en esta población, así como de su conectividad por carretera y ferrocarril. Por su parte, el tercer apartado está dedicado a los datos y la metodología utilizados a partir 
de los cuales obtener los resultados. Unos resultados que se muestran en el apartado cuatro, dividido a su vez en dos subapartados. En el primero de ellos se indican las características principales de la muestra objeto de estudio y en el segundo se analizan los resultados sobre la movilidad de los estudiantes del Campus Vila-seca (objetivo 1). En el quinto apartado se definen y desarrollan propuestas para alcanzar una movilidad más sostenible (objetivo 2).

Figura 1. Localización del Campus Vila-seca de la URV en la provincia de Tarragona

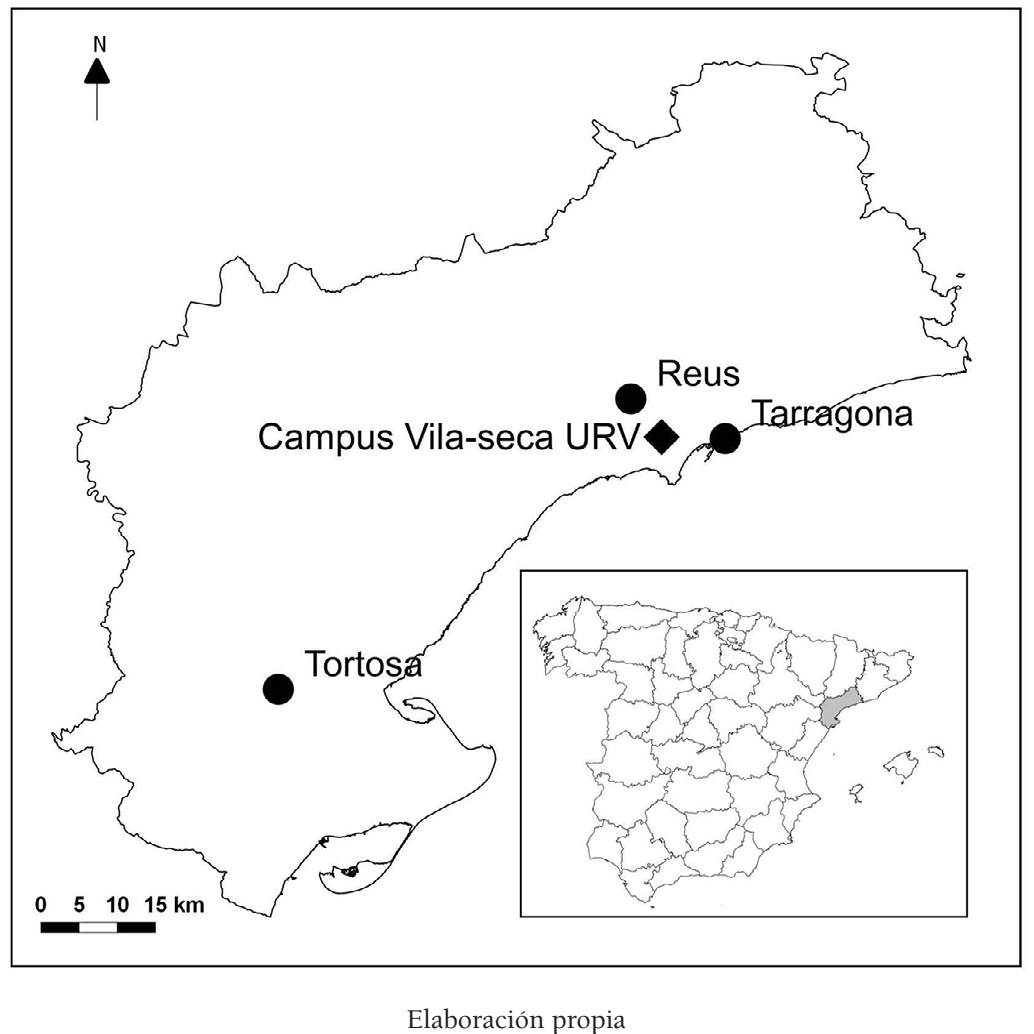

\section{VILA-SECA Y EL CAMPUS UNIVERSITARIO DE LA URV: LOCALIZACIÓN Y CONECTIVIDAD}

El campus Vila-seca de la URV se encuentra situado en el borde meridional del perímetro urbano de la localidad que le da nombre. Junto al centro universitario hay dos institutos de estudios secundarios y diversos equipamientos deportivos de titularidad municipal. Los terrenos del campus constan de una explanada utilizada como aparcamiento de acceso libre y que también es utilizada por los usuarios de los equipamientos deportivos anteriormente mencionados. El aparcamiento ha sido remodelado completamente recientemente. También es de acceso libre el aparcamiento situado junto a los dos centros de estudios secundarios y que se encuentra a poco menos de 250 metros del edificio de la Facultad de Turismo y Geografía. A una distancia similar se encuentra la Vía Máxima donde hay la posibilidad de aparcar tanto en batería como en paralelo. En la calle que da acceso al centro universitario (c. Joanot Martorell) actualmente también está permitido aparcar.

El municipio de Vila-seca se encuentra en la provincia de Tarragona, a una distancia inferior a los 10 km tanto de la ciudad de Tarragona en dirección oeste, como de Reus, en dirección sureste. Por su término municipal transcurren importantes vías de comunicación. En dirección noreste-suroeste la autopista AP-7 y la autovía A-7. La primera discurre por el norte del núcleo urbano y su salida/entrada número 35 se encuentra a unos 2,5 km del campus universitario. Por su parte la A-7 bordea el núcleo urbano por el sur y su salida/entrada 1153 está situada a poco menos de $1 \mathrm{~km}$ del campus (Figura 2). Esta vía de comunicación rápida sin peaje funciona como circunvalación, evitando que los vehículos en tránsito tengan que cruzar Vila-seca, tal y como sí sucedía cuando todo el tráfico pasaba por la carretera N-340. El traza- 
do de esta carretera aún comunica Vila-seca y Tarragona a través de los barrios de poniente de la capital provincial. Por el oeste del núcleo urbano se encuentra la carretera C-14 que comunica Reus con Salou.

Figura 2. Núcleo urbano de Vila-seca con la localización del Campus de la URV, la estación de ferrocarril y la parada de autobuses más cercana al centro universitario

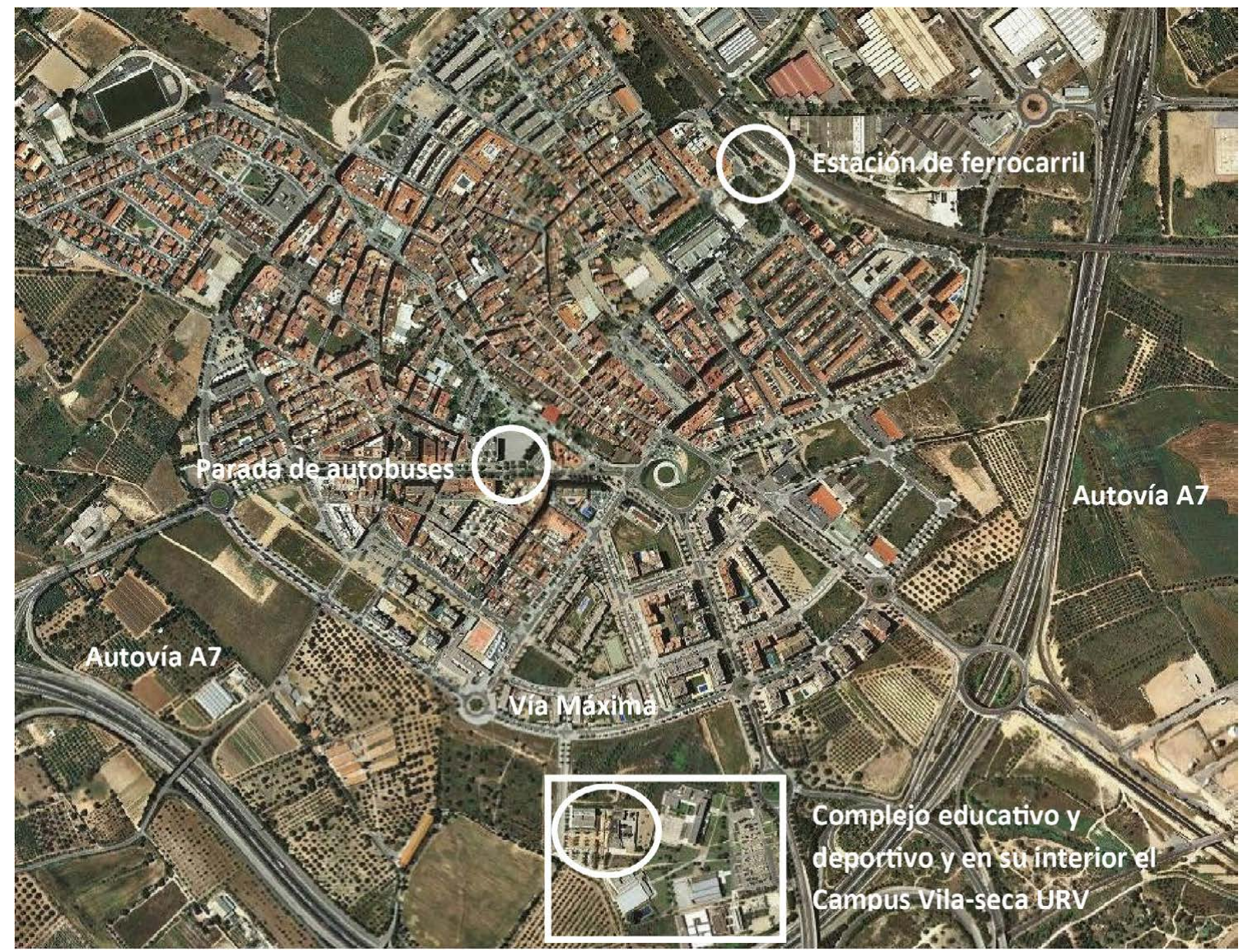

Fuente: Institut Cartogràfic de Catalunya (ICC). Elaboración propia

Algunas de estas vías de comunicación son utilizadas por los autobuses que, a través de diferentes líneas regulares, conectan Vila-seca con Cambrils, Reus, Salou y Tarragona, sin necesidad de realizar trasbordo alguno. La distancia entre la parada de autobús y el Campus Vila-seca es de aproximadamente $1 \mathrm{~km}$. La mayor frecuencia se da con Tarragona (más de 30 en cada sentido), puesto que todos los que enlazan Vila-seca con Cambrils y algunos de los de Salou tienen su origen o destino en Tarragona. En cambio sólo hay 14 autobuses que unen Vila-seca con Reus, la mitad en cada sentido.

En el extremo septentrional del núcleo urbano de Vila-seca se encuentra la estación de tren que actualmente funciona como apeadero (Figura 2). Se trata de la línea de ferrocarril que une Barcelona y Reus, pasando por Tarragona. Desde Reus la línea se bifurca en dirección Lleida o Móra la Nova. La distancia de la estación hasta la Facultad de Turismo y Geografía es de $1,5 \mathrm{~km}$ en un recorrido por el entramado urbano. En el momento de realizar el estudio, en días laborables en Vila-seca tenían parada dieciséis trenes procedentes de Barcelona, más otros dos con origen en Tarragona. Todos tienen parada en Reus y siete finalizan allí su recorrido, seis llegan como mínimo hasta Móra la Nova (uno con destino en Madrid) y cinco hasta La Plana de Picamoixons de los cuales tres siguen el recorrido hasta Lleida. Por su parte, diecisiete convoyes tienen parada en Vila-seca procedentes de Reus (en seis casos es el origen). Todos ellos con destino final Barcelona a excepción de dos que terminan su recorrido en Tarragona. De los once trenes que no tienen su inicio en Reus, seis vienen de Móra la Nova (uno con origen en Madrid), tres tienen su origen en Lleida y dos en La Plana-Picamoixons. Desde el año 2014 la introducción de la línea de cercanías entre Tarragona y Reus ha provocado que la frecuencia de trenes con parada en Vila-seca haya aumentado significativamente. 


\section{DATOS Y METODOLOGÍA}

Los resultados de este estudio son fruto de una encuesta realizada durante los meses de marzo y abril de 2013, es decir, una vez iniciado el segundo semestre del curso académico 2012-2013. Los encuestados fueron alumnos de primer, segundo y tercer curso del Grado en Turismo y del Grado en Geografía y Ordenación del Territorio de la URV. No han participado los alumnos de cuarto curso debido a que su presencia en las aulas en esas fechas es mucho menor debido a la realización de las prácticas externas curriculares y del trabajo final de grado.

Los alumnos respondieron la encuesta en el aula ordinaria, para lo cual solicitamos a los profesores responsables de una materia obligatoria de los respectivos grados y cursos disponer de la última media hora de clase En el caso de los alumnos de primero se realizó aprovechando una materia común para ambos grados. El objetivo de hacer la encuesta a inicios del segundo cuatrimestre, en el aula ordinaria y en materias obligatorias era obtener una muestra elevada. No obstante, a pesar de estas premisas, los estudiantes encuestados fueron 191, cifra que representa un 58,6\% del total de la población que se pretende caracterizar: 326 alumnos de $1^{\circ}, 2^{\circ}$ y $3^{\circ}$ curso (223 del grado de Turismo y 103 del grado de Geografía y Ordenación del Territorio). A pesar de todo, la muestra tiene un nivel de confianza del $95 \%$ con un margen de error del 5\%.

El cuestionario consta de 18 preguntas. La gran mayoría presentan un abanico de respuestas posibles, aunque también las hay de respuesta numérica y binaria (sí o no). Únicamente hay una pregunta de respuesta abierta y otra de tipo Likert. Esta última pregunta es clave para conocer la predisposición de los estudiantes que no utilizan el transporte público a cambiar: ¿cómo de cierto es que cambiarían a un medio de transporte colectivo si las razones aducidas para no hacerlo no se dieran? Siendo 0 nada cierto y 10 totalmente cierto. Que los estudiantes tengan disponible un medio de transporte colectivo para realizar los desplazamientos y que los factores que explican que no lo utilicen desaparezcan no significa necesariamente que vayan a utilizarlo. El objetivo de esta pregunta es obtener de cada estudiante que se desplaza en coche el porcentaje o fracción potencial de cambio a un medio transporte público si las razones que lo impiden no existieran, cosa que permitirá medir la contribución del total de la muestra en base a la suma de las fracciones individuales. De esta manera se evita perder las contribuciones individuales tal y como sí sucede en el caso de la clasificación binaria.

\section{RESULTADOS}

A continuación se muestran los resultados obtenidos en relación a la movilidad de los estudiantes que se desplazan hasta el Campus Vila-seca de la URV, seguidos por un conjunto de propuestas para intentar reducir la movilidad de los estudiantes en coche. Previamente, se presenta una descripción general en relación a los rasgos principales de los encuestados.

\subsection{Características principales de la muestra}

La distribución por sexo de los 191 estudiantes que forman el conjunto de la muestra presenta valores muy similares, aunque hay una ligera mayor presencia de mujeres que de hombres (53,4\% y $49,6 \%$, respectivamente). En cambio sí que existen diferencias significativas si se analiza está variable teniendo en cuenta qué están estudiando. La distribución es totalmente opuesta. Un 75,2\% de los alumnos del Grado en Turismo encuestados son mujeres, mientras que un 78,2\% de los alumnos del Grado en Geografía y Ordenación del Territorio son hombres. En relación a la edad, la muestra se ha dividido entre aquellos que tienen como máximo 25 años y aquellos que ya han superado esta edad. En el primer grupo se encuentra el 80,1\% de la muestra, es decir, 4 de cada 5.

Teniendo en cuenta los estudios cursados, un total de 113 encuestas corresponden a estudiantes de Turismo y las 78 restantes a estudiantes de Geografía y Ordenación del Territorio. La encuesta fue realizada por 2 de cada 4 estudiantes de Turismo y por 3 de cada 4 de Geografía y Ordenación del Territorio. Mientras que en el conjunto de estudiantes de los tres primeros cursos solo un 31,6\% estudian Geografía y Ordenación del Territorio, cabe indicar que en la muestra analizada representan un 40,8\%. Un 35,6\% de los estudiantes manifestaron que tenían las asignaturas mayoritariamente por la mañana, un $37,7 \%$ por la tarde y el restante $26,7 \%$ tanto por la mañana como por la tarde. 
Figura 3. Distribución de los estudiantes según el municipio de residencia durante el curso académico

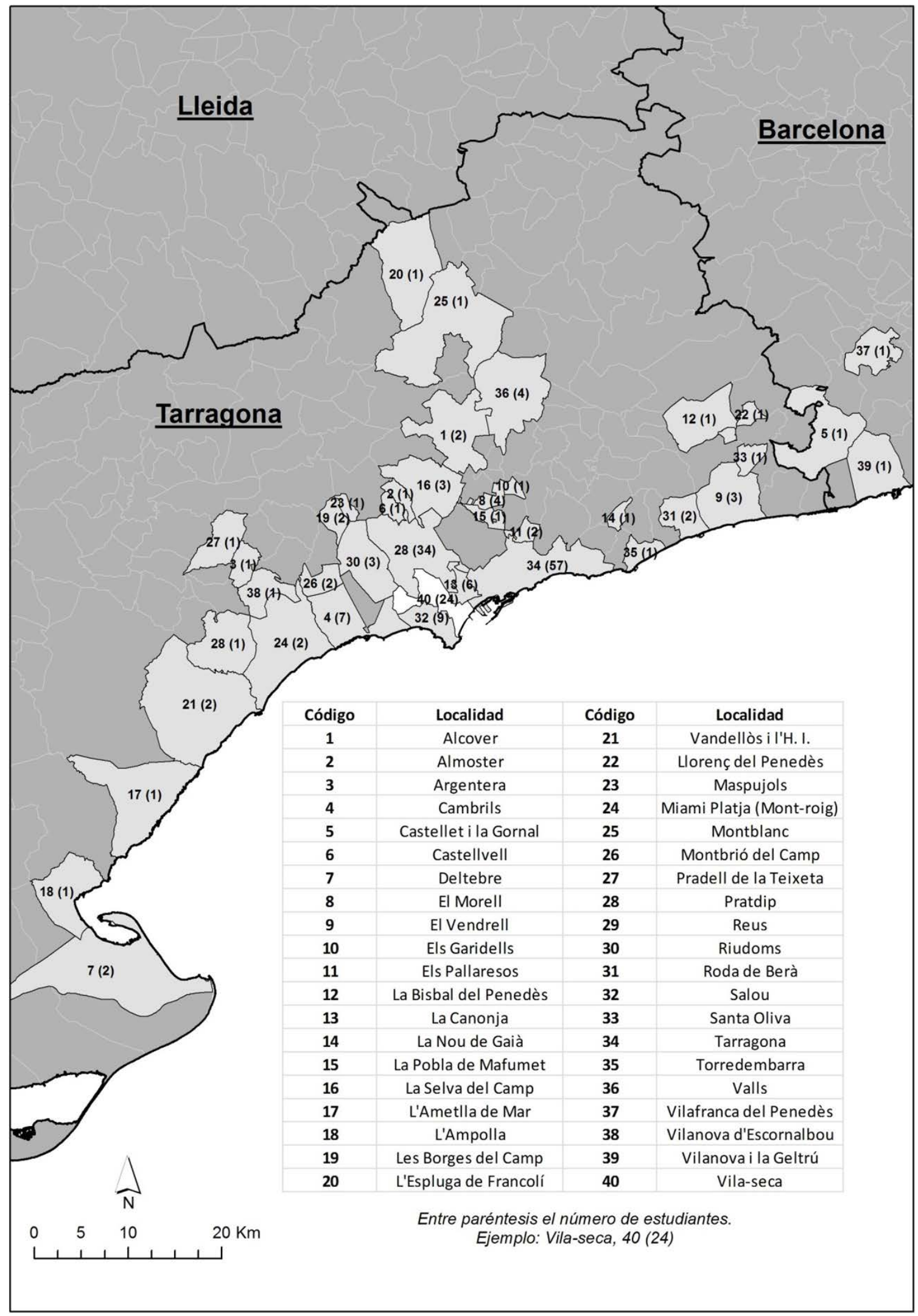

Elaboración propia 
La Tabla 1 muestra la distribución de los encuestados teniendo en cuenta el curso académico y los estudios que están realizando. Un 39,3\% del total están en primer curso, un 31,4\% en segundo y un $29,3 \% \%$ en tercero. En ambos grados el mayor número también corresponde a los estudiantes de primer curso. Un $40,7 \%$ en Turismo y un 37,2\% en Geografía y Ordenación del Territorio.

Tabla 1. Estudiantes según grado y curso

\begin{tabular}{|l|c|c|c|}
\hline & 1r curso & $2^{\circ}$ curso & 3 r curso \\
\hline Total & $39,3 \%$ & $31,4 \%$ & $29,3 \%$ \\
\hline Turismo & $40,7 \%$. & $31,9 \%$ & $27,4 \%$ \\
\hline Geografía y O.T. & $37,2 \%$ & $30,8 \%$ & $32,0 \%$ \\
\hline
\end{tabular}

Elaboración propia

El lugar de residencia durante el curso académico es un factor clave en relación a la movilidad de los estudiantes ya que condiciona tanto la distancia como la existencia de más o menos alternativas para desplazarse hasta el centro universitario. La figura 3 muestra la distribución de los alumnos según el municipio de residencia. Un total de 40 localidades están representadas. La gran mayoría están situadas en la provincia de Tarragona (92,5\%), aunque también hay 3 municipios de la provincia de Barcelona (Castellet i la Gornal, Vilafranca del Penedès y Vilanova i la Geltrú). Un 98,4\% de los estudiantes encuestados residen durante el curso en municipios de la provincia de Tarragona.

Únicamente en tres municipios residen el 60,2\% de los estudiantes: 57 en Tarragona, 34 en Reus y 24 en Vila-seca. Se trata de las dos principales ciudades de la provincia y la localidad donde se encuentra la Facultad de Turismo y Geografía. Ninguna otra localidad llega a la cifra de 10 estudiantes encuestados. Salou está en cuarta posición con 9. Una concentración en tres municipios que aumenta en el caso de los estudiantes de Turismo hasta un $64,6 \%$, más de 10 puntos por encima que entre los estudiantes de Geografía y Ordenación del Territorio (53,8\%).

La tabla 2 muestra la distribución de los estudiantes teniendo en cuenta la distancia desde su localidad de residencia durante el curso hasta el centro universitario en Vila-seca. Como se puede observar, más del $80 \%$ residen a una distancia que no supera los $20 \mathrm{Km}$. Teniendo en cuenta la información facilitada en el apartado 2, un total de 120 alumnos podrían llegar desde su localidad de residencia hasta Vila-seca ya sea en autobús o en tren sin necesidad de transbordo. Es decir, el 62,8\% de los estudiantes encuestados tienen la posibilidad de utilizar un medio de transporte público colectivo. A estos hay que añadir los 24 que residen en Vila-seca y que podrían realizar el trayecto hasta la Facultad de Turismo y Geografía a pie o en bici. Vila-seca dispone de carril bici en buena parte del núcleo urbano. Un carril bici que tiene continuación hasta Salou. En definitiva, más de tres cuartas partes no tendrían por qué utilizar el vehículo privado.

La gran mayoría de los estudiantes encuestados viven con sus padres (67\%), seguidos por aquellos que comparten piso con otros estudiantes $(13,6 \%)$, con su propia familia $(7,9 \%)$, solos $(5,2 \%)$, con otros familiares que no sean los padres, pareja o hijos $(5,2 \%)$ o en una residencia de estudiantes $(1,1 \%)$.

Tabla 2. Distribución de los estudiantes teniendo en cuenta la distancia desde la localidad de residencia hasta la Facultad de Turismo y Geografía

\begin{tabular}{|c|c|c|}
\hline Distancia & Estudiantes & Frecuencia \\
\hline Hasta $10 \mathrm{Km}$. & 130 & $68,1 \%$ \\
\hline $10,1-20 \mathrm{Km}$. & 27 & $14,1 \%$ \\
\hline $20,1-40 \mathrm{Km}$. & 20 & $10,5 \%$ \\
\hline $40,1-60 \mathrm{Km}$. & 10 & $5,2 \%$ \\
\hline Más de $60 \mathrm{Km}$. & 4 & $2,1 \%$ \\
\hline \multicolumn{3}{|c|}{ Elaboración propia }
\end{tabular}

\subsection{La movilidad de los estudiantes}

De acuerdo con la información facilitada en el apartado anterior, únicamente 47 estudiantes de los que no residen en Vila-seca no disponen de conexión directa de autobús o tren. No obstante, los resul- 
tados de la encuesta muestran que 123 de los 191 estudiantes encuestados $(64,4 \%)$ se desplazan habitualmente desde su residencia durante el curso académico hasta la Faculta de Turismo y Geografía en coche. No hay diferencias significativas por lo que respecta al sexo de los estudiantes que se desplazan en coche. Un $51,2 \%$ son mujeres, cifra similar a su representación en el conjunto de la muestra (53,4\%). La gran mayoría de los estudiantes que se desplazan en coche hacen el trayecto solos (67,5\%). Las razones principales que indican para no compartir coche son que no conocen a nadie de su misma zona o zonas próximas y que de conocerlos no cuentan con horarios compatibles.

A mucha distancia del coche se encuentra el autobús, utilizado por un 19,9\% de los estudiantes (potencialmente un 56\%). En tercer lugar está el tren con un 7,3\% (potencialmente un 54,5\%), seguidos de un $6,8 \%$ que van a pie y un $1,1 \%$ en moto. Un alumno, residente en Vila-seca, manifestó que se desplaza habitualmente en patinete (hecho que ha sido comprobado por los autores de este estudio), mientras que ninguno se desplaza en bicicleta. Un estudio publicado recientemente en relación a la movilidad en el Campus Terres de l'Ebre de la URV, situado en Tortosa, indica que un $73 \%$ de los estudiantes se desplazan en coche, un $23 \%$ a pie, un $2 \%$ en moto, un 1\% en autobús y un 1\% en bicicleta (Mcrit, 2013).

La figura 4 muestra el medio de transporte utilizado por los estudiantes teniendo en cuenta la localidad donde residen. Como se puede observar, se han agrupado los estudiantes de Salou y Cambrils y también los del resto de localidades. En Vila-seca predominan aquellos estudiantes que hacen el recorrido a pie $(54,2 \%)$, aunque más de un tercio $(37,5 \%)$ se desplazan en coche. Esta cifra es la más baja de los desplazamientos en coche por ciudades, pero es muy elevada si tenemos en cuenta que son estudiantes que residen en la misma localidad donde se encuentra el centro universitario. En el caso de Tarragona un $40,4 \%$ de los estudiantes se desplazan en automóvil privado. No obstante, hay que hacer notar que, como en Vila-seca, tampoco es el grupo más importante. Un 52,6\% viajan en autobús. Sólo un 7\% utilizan el tren. La frecuencia de autobuses entre Tarragona y Vila-seca es mayor que la de trenes y a su vez, la distancia entre la parada de autobús y el Campus Vila-seca es menor que la existente con la estación de tren.

Figura 4. Medio de transporte utilizado por los estudiantes en relación a la localidad de residencia durante el curso académico

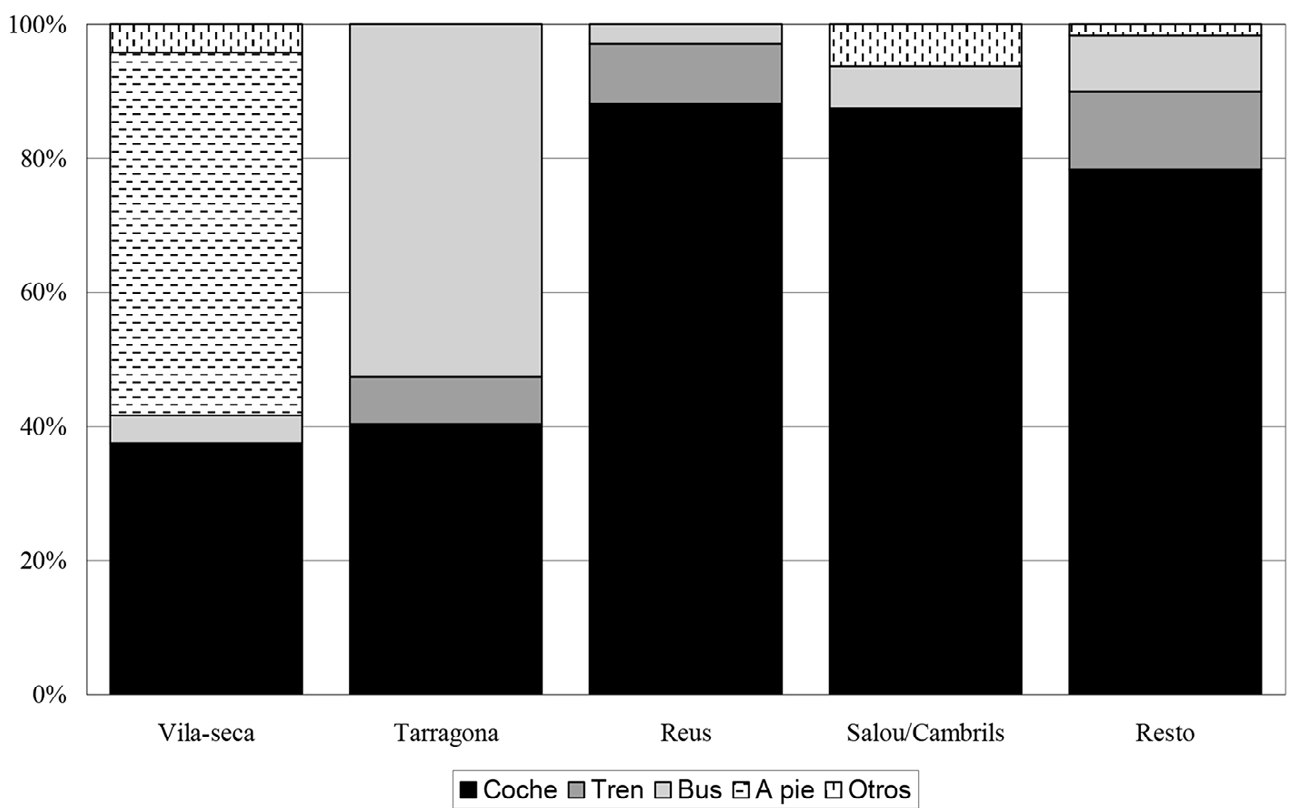

Elaboración propia

Tanto los estudiantes que residen en Reus como en Salou/Cambrils utilizan mayoritariamente el coche. En el primer caso representan casi un 90\% del total de estudiantes de Reus, siendo un 87,5\% en Salou y Cambrils. A pesar de existir conexión con autobús, sólo un 8,3\% de los estudiantes de estas dos últimas localidades utilizan este medio de transporte en su desplazamiento hasta Vila-seca. Una cifra menor en el caso de Reus con únicamente un 2,9\% en autobús, mientras que sólo un $8,8 \%$ hacen el viaje en tren. 
Finalmente, un 78,3\% de los 60 estudiantes que residen en las otras 35 localidades se desplazan en coche. Una cifra que coincide con la de aquellos que no disponen de conexión directa en tren con Vila-seca, aunque son dos segmentos diferentes. Un $11,7 \%$ utilizan el tren y un 8,3\% el autobús. Hay algunos alumnos que disponen de conexión directa en tren pero utilizan el coche y en cambio hay otros que se desplazan en vehículo privado hasta estaciones de tren con conexión con Vila-seca, ya sea conducido por ellos mismos o por algún familiar. Lo mismo sucede en el caso de los que utilizan el autobús.

Si se analiza la distribución de los alumnos teniendo en cuenta el medio de transporte utilizado y los estudios cursados, un $61,1 \%$ de los de Turismo y un 69,2\% de los de Geografía y Ordenación del Territorio se desplazan en coche (Figura 5). Se puede observar que la diferencia más importante entre los alumnos de ambos grados se produce entre aquellos que utilizan el autobús, un $24,8 \%$ de los estudiantes de Turismo y sólo un 12,8\% de Geografía y Ordenación del Territorio. El análisis por cursos muestra como en primero y en tercero los alumnos que utilizan el coche representan casi un $70 \%$ mientras que en segundo son poco más del 55\%. En los alumnos de segundo curso destacan aquellos que se desplazan en autobús $(28,2 \%)$.

Figura 5. Medio de transporte utilizado en relación a los estudios y el curso

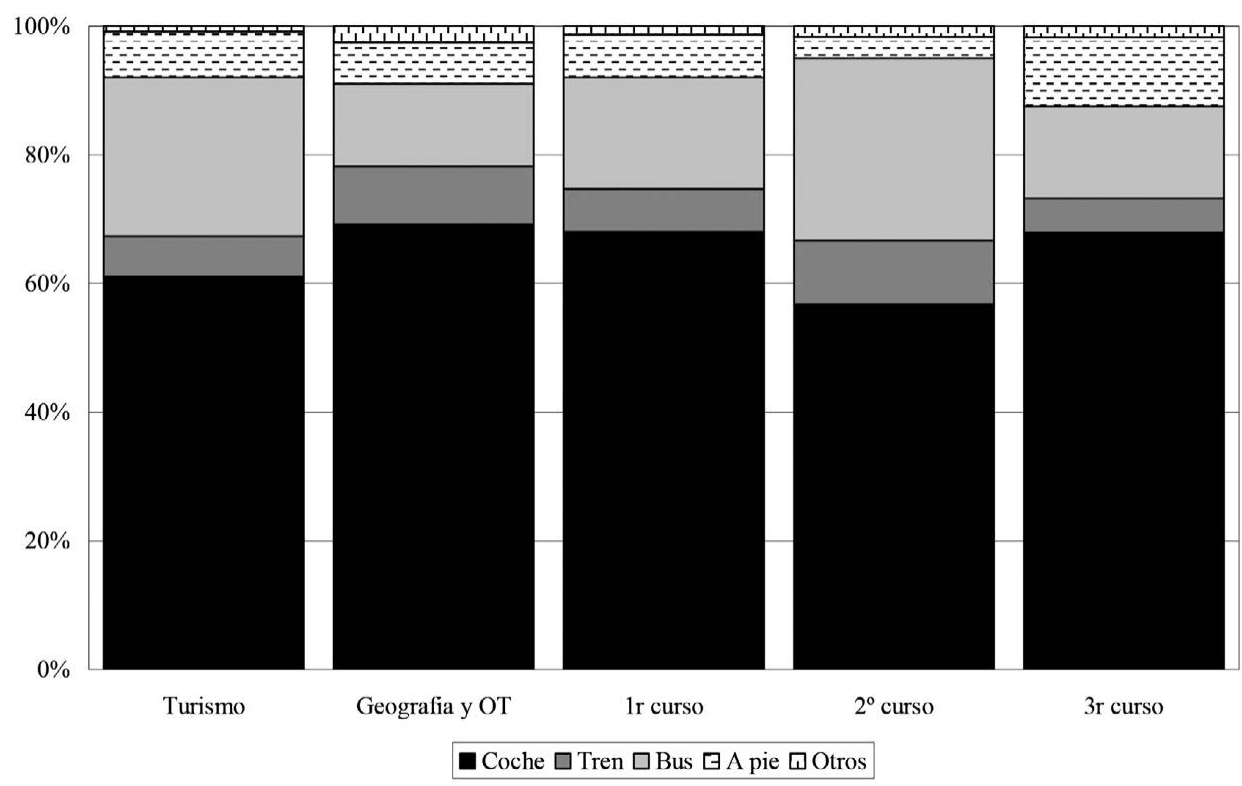

Elaboración propia

Los principales motivos por los cuales los estudiantes se desplazan en coche o en tren/autobús hasta el centro universitario difieren de manera importante, tal y como se puede ver en la tabla 3. En el primer caso, destacan aquellos que justifican el hecho de desplazarse en coche por la rapidez con un $41,5 \%$. Por su parte, un $26 \%$ lo atribuyen a la poca oferta de transporte público, mientras que un 20,3\% dice ser debido a la comodidad del viaje en coche. Estas tres razones aglutinan casi un 90\% del total de estudiantes que utilizan el vehículo privado. Si se compara con el estudio ya comentado realizado en el Campus Terres de l'Ebre de la URV, un 29\% dijeron que la razón principal de la elección del coche es la comodidad, un $23 \%$ la rapidez y un $11 \%$ el hecho que el transporte público no era una alternativa. En el caso del centro universitario de Tortosa hay que destacar que un $11 \%$ manifestaron que la razón principal era el precio.

En el caso de los estudiantes que viajan en tren o en autobús, también únicamente tres razones concentran la mayor parte de las respuestas (79,3\%). El principal motivo de viajar en transporte público colectivo es el precio $(32,1 \%)$, seguido por la no disponibilidad de vehículo privado $(28,3 \%)$ y en tercer lugar por la no disponibilidad de licencia de conducir. Es decir, hay la posibilidad de que un $47,1 \%$ de los estudiantes que actualmente viajan en tren o autobús cambien al coche en el caso que lleguen a disponer de uno y/o obtengan la licencia de conducir. En una escala de 0 a 10, la valoración del servicio de autobús por parte de aquellos estudiantes que utilizan este medio de transporte es, en promedio, un 3,2. Una cifra algo superior, pero también muy baja, en la valoración del tren: 4,7. 
Tabla 3. Motivo principal de la elección del coche o del tren/bus como medio de transporte desde la residencia hasta el Campus Vila-seca

\begin{tabular}{|l|c|c|}
\hline \multicolumn{1}{|c|}{ Motivo de la elección } & Coche & Tren/Bus \\
\hline Rapidez en el desplazamiento & $41,5 \%$ & $0,0 \%$ \\
\hline Poca oferta de transporte público & $26,0 \%$ & - \\
\hline Comodidad & $20,3 \%$ & $0,0 \%$ \\
\hline Precio & $0,0 \%$ & $32,1 \%$ \\
\hline No disponibilidad de vehículo privado & - & $28,3 \%$ \\
\hline Proximidad al lugar de residencia & $0,8 \%$ & $9,4 \%$ \\
\hline No disponibilidad de licencia de conducir & - & $18,9 \%$ \\
\hline Combinación adecuada de horarios & $4,9 \%$ & $1,9 \%$ \\
\hline Puntualidad & $4,1 \%$ & $0,0 \%$ \\
\hline Otros & $2,4 \%$ & $9,4 \%$ \\
\hline
\end{tabular}

Elaboración propia

Por ciudades, es en Salou donde los estudiantes que viajan en coche manifiestan de manera más clara $(77,8 \%)$ que la razón principal es la rapidez en el desplazamiento, seguidos por los de Vila-seca con un $55,6 \%$. En el caso de Reus, Tarragona y "resto de localidades" los valores son 43,3\%, 39, $1 \%$ y 34\%, respectivamente. Finalmente, representan un 20\% en Cambrils. En el caso de la capital provincial un 34,8\% manifestaron que el motivo de viajar en coche era la comodidad y un 13\% la poca oferta de transporte público. Esta razón fue argüida por un 26,7\% de los estudiantes de Reus y un 42,5\% de los del "resto de localidades" en donde no existe conexión directa en autobús con Vila-seca.

Los estudiantes que se desplazan en coche y que residen en Tarragona, Reus, Salou o Cambrils (67) fueron preguntados sobre cuál era la principal razón para no viajar en transporte público colectivo. Un $52,2 \%$ dijeron que era por los horarios y un $20,9 \%$ por el recorrido del viaje. Otras respuestas, todas ellas inferiores al $10 \%$, fueron por considerar que la calidad del servicio es deficiente o por un precio excesivo. En el caso de Reus los horarios son la razón principal de un 70\% de los que viajan en coche. Motivo que sólo representa un 34,8\% en el caso de los estudiantes que residen en Tarragona. En el caso de los 47 estudiantes que se desplazan en coche y que residen en "resto de localidades", la gran mayoría indicaron que era debido o bien a que no existía transporte público colectivo, al horario en caso de existir (trenes) o a que el recorrido a realizar hasta la localidad más próxima donde sí que existe conexión directa no se ajusta a sus necesidades. También en algunos casos la razón aducida era el precio excesivo que resulta de coger más de un transporte público, es decir, viaje con trasbordo.

Un análisis de las horas de llegada y de salida de trenes y autobuses en Vila-seca, teniendo en cuenta los horarios de los estudiantes, muestra que los servicios de ferrocarril procedentes tanto de Móra la Nova como de Alcover no se ajustan en su conjunto al horario académico. Cambrils sí que tiene buena conexión con Vila-seca en autobús en horario de mañana. Los estudiantes pueden tomar el que llega a las 8:55 h, aunque puede suponer no empezar puntuales la primera sesión de clase. Disponen de un autobús de regreso a las $13: 27 \mathrm{~h}$ y a las $14: 17 \mathrm{~h}$, adecuados en el caso de finalizar clases a las $13 \mathrm{o}$ a las 14 horas, respectivamente. El horario es menos propicio por la tarde, especialmente si son alumnos que empiezan a las 15 h. Los horarios de los autobuses entre Salou y Vila-seca son relativamente adecuados tanto en horario de mañana como de tarde. Por la mañana hay un autobús que llega a Vila-seca a las 8:35 y otro que regresa a Salou a las 13:27. Por la tarde hay uno que llega a las $14.45 \mathrm{~h}$ y otro que regresa a las 19:15 h. La proximidad de Cambrils y de Salou con Vila-seca explicarían la utilización mayoritaria del coche privado a pesar de una más que aceptable conectividad en bus. También habría que tener en cuenta la mayor o menor distancia de la residencia de los estudiantes hasta la parada de autobuses en Cambrils y en Salou.

No es de extrañar que la mayoría de estudiantes de Reus viajen en coche (90\%) y que un $70 \%$ lo atribuyan a los horarios del trasporte público. El primer autobús que llega a Vila-seca procedente de Reus lo hace a las 9:30 h, tarde si las clases empiezan a las 9 de la mañana. En cambio, el tren, o bien llega con más de 1 hora de antelación al inicio de las clases o bien llega casi media hora tarde, con el añadido que la estación de ferrocarril se encuentra a unos 15 minutos de la Facultad de Turismo y Geografía. Los alumnos que tienen horario de tarde tienen mejor combinación, puesto que hay un tren que llega a Vila-seca a las 
14.46 h. No obstante, el problema puede estar en el momento de regresar. Así pues, la poca relación entre horario académico y horario de los medios de transporte y la proximidad entre Vila-seca y Reus explican el uso masivo del automóvil por parte de los estudiantes residentes en Reus. A su vez, también hay que tener en cuenta que la distancia desde las viviendas de los estudiantes hasta la estación de autobuses o la estación de ferrocarril de Reus puede variar significativamente.

Tarragona presenta una muy buena conexión con Vila-seca en transporte público, especialmente por medio del autobús. No obstante, un $40,4 \%$ de los estudiantes se desplaza en coche. Como en el caso de Reus, la proximidad con Vila-seca y la distancia que pueda haber entre las viviendas de los estudiantes y la estación de autobuses o la estación de ferrocarril de Tarragona juega un papel importante.

Finalmente, se preguntó a los 114 estudiantes que se desplazan en coche y que no residen en Vila-seca si cambiarían a un medio de transporte colectivo si las razones aducidas para no hacerlo no se dieran (respuesta binaria: sí o no) y, a continuación, que calificaran de 0 a 10 cómo de cierto era que cambiarían a un medio de transporte colectivo si las razones aducidas para no hacerlo no se dieran, siendo 0 nada cierto y 10 totalmente cierto. El objetivo de esta segunda pregunta es obtener para cada uno de los estudiantes de este segmento el porcentaje o fracción del potencial cambio de coche a transporte público en su desplazamiento hasta la Facultad de Turismo y Geografía, cosa que permitirá medir la contribución del total de la muestra en base a la suma de las fracciones individuales. De esta manera se evita perder las contribuciones individuales tal y como sí sucede en el caso de la clasificación binaria.

Tabla 4. Porcentaje de estudiantes que viajan en coche y que cambiarían a un medio de transporte colectivo si no se dieran las circunstancias que hacen que no lo tomen

\begin{tabular}{|l|c|c|}
\hline \multicolumn{1}{|c|}{ Estudiantes según localidad de residencia (N) } & Respuesta binaria & Respuesta numérica \\
\hline Todos excepto residentes en Vila-seca (114) & $52,6 \%$ & $62,2 \%$ \\
\hline Localidades con conexión directa en bus (67) & $50,7 \%$ & $61,0 \%$ \\
\hline Tarragona (23) & $60,9 \%$ & $63,5 \%$ \\
\hline Reus (30) & $50,0 \%$ & $64,7 \%$ \\
\hline Localidades sin conexión directa en bus (47) & $55,3 \%$ & $63,8 \%$ \\
\hline
\end{tabular}

Elaboración propia

La tabla 4 muestra el porcentaje de estudiantes que cambiarían el coche como medio de transporte para desplazarse hasta el campus universitario por uno de colectivo teniendo en cuenta la localidad de residencia y diferenciando entre el resultado de la pregunta con respuesta binaria y de la que permite una repuesta numérica entre 0 y 10 . En el primer caso (columna central) el porcentaje más elevado corresponde a los alumnos residentes en Tarragona $(60,9 \%)$, mientras que el más bajo se da entre los estudiantes de Reus (50\%).

Como se puede observar, la suma de las fracciones individuales (columna de la derecha) presenta valores superiores a los obtenidos en la pregunta con respuesta binaria en todos los casos. El aumento más importante (más de 14 puntos) se da entre los estudiantes procedentes de Reus. Un 64,7\% cambiaría a un medio de transporte colectivo si se tienen en cuenta la suma de las fracciones individuales. Por su parte, el porcentaje menor se da entre el conjunto de estudiantes que viajan en coche y residen en localidades con conexión directa en autobús con Vila-seca (61\%). Diferenciando entre estudiantes del grado en Turismo y el grado en Geografía y Ordenación del Territorio, el porcentaje que estaría dispuesto a cambiar el coche por un medio de transporte colectivo es, respectivamente, un $57,7 \%$ y un $68 \%$.

La figura 6 muestra la distribución de las respuestas de los 67 estudiantes que viajan en coche y residen en Tarragona, Reus, Cambrils o Salou a la pregunta numérica (0-10) sobre si cambiarían a transporte colectivo. Sólo 6 contestaron 0, es decir, no cambiarían de ninguna manera a un medio de transporte colectivo. Por su parte 9 estudiantes dijeron que sería totalmente cierto el cambio (10). Los restantes 52 añaden matices, aunque 30 de ellos contestaron 7,8 o 9 . Valores que podemos atribuir a un calificativo de "bastante cierto". La suma de las contribuciones individuales da un incremento en la probabilidad de utilizar el transporte público del 61\% (Tabla 4), es decir, 41 estudiantes. De esta manera el porcentaje de estudiantes que se desplazan en coche pasaría de un $64,4 \%$ a un $42,9 \%$ del total. Una cifra que aún se podría reducir más si se implementara un conjunto de estrategias para que un mayor número de estudiantes que residen en Vila-seca y en el resto de localidades no cojan el coche. 
Figura 6. Distribución de las respuestas a la pregunta sobre el potencial cambio de desplazamiento en coche a transporte colectivo

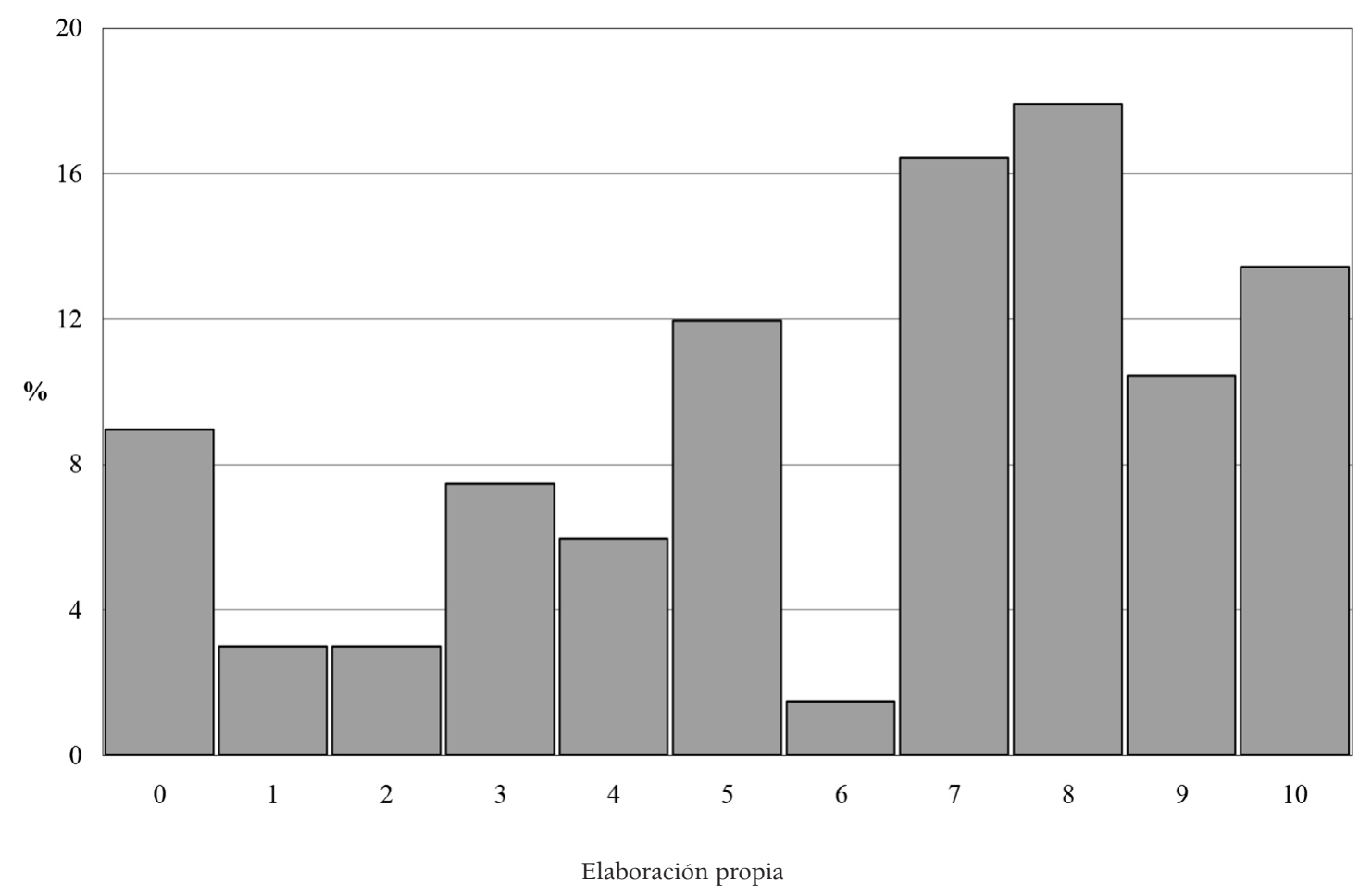

\section{PROPUESTAS PARA ALCANZAR UNA MOVILIDAD MÁS SOSTENIBLE}

A continuación se muestran las propuestas para reducir el uso del coche como medio de transporte por parte de los estudiantes del Campus Vila-seca y para alcanzar una movilidad más sostenible. En cada una de las propuestas se indican los agentes implicados, la prioridad en llevarla a cabo y la facilidad en su aplicación.

\section{a) Sesión informativa con los estudiantes.}

El objetivo de esta sesión es divulgar entre los estudiantes la existencia del Plan de Medio Ambiente de la URV y de sus cinco líneas estratégicas, haciendo mayor hincapié en la segunda centrada en la movilidad. Así pues, hay que informar a los estudiantes sobre el abanico de posibilidades para desplazarse en transporte público colectivo (trayectos, frecuencia y horarios) hasta el Campus Vila-seca y del precio especial que se aplica a los miembros de la comunidad universitaria en el caso del autobús. En relación al tren, la gran mayoría de estudiantes pueden acogerse al descuento asociado al Carnet Jove (<30 años). En el caso de los estudiantes que residen en Vila-seca hay que incidir en el desplazamiento a pie o bien en bicicleta. Además de la información referente a aquellas localidades con conexión directa también hay que hacer mención a la posibilidad de utilizar el apeadero situado cerca de Port Aventura y que forma parte de la línea entre Barcelona y Tortosa. El apeadero se encuentra al lado de la Avenida Alcalde Pere Molas, que une Salou y Vila-seca, por donde pasa el trazado de un carril bici. La Facultad de Turismo y Geografía se encuentra a unos 2,5 Km, con lo cual también sería posible realizar este trayecto a pie. Aunque los horarios de los trenes no son adecuados en según qué franjas horarias, es una opción a tener en cuenta, siempre y cuando se cumplan unas mínimas condiciones de seguridad y que el Campus Vila-seca disponga de una adecuada zona de aparcamiento de bicicletas.

Otro aspecto interesante es el de informar a los estudiantes de la posibilidad de desplazarse en coche hasta estaciones de trenes cercanas a su lugar de residencia, donde pueden estacionarlo de manera gratuita sin excesivos problemas de espacio y, de esta manera, viajar en tren hasta Vila-seca. Dos ejemplos claros son las estaciones de Sant Vicenç de Calders y de Altafulla, que pueden dar este servicio a los alumnos residentes, respectivamente, en la comarca de El Baix Penedès y en las poblaciones situadas en la parte oriental de la comarca de El Tarragonès. 
La posibilidad que los estudiantes adquieran como hábito la utilización del transporte público será mayor cuanto antes reciban esta información, por lo cual se propone que esta sesión tenga lugar a inicios del curso académico. En el caso de los estudiantes de primer curso la información se podría facilitar en el marco de la sesión de acogida durante la cual son informados sobre aspectos generales del funcionamiento del centro universitario y de sus servicios. En el caso del resto de estudiantes, la sesión se podría impartir aprovechando alguna asignatura relacionada con las cuestiones de carácter ambiental o de sostenibilidad presentes tanto en el Grado en Turismo como en el grado en Geografía y Ordenación del Territorio. Por ejemplo, en el primer cuatrimestre de 3r curso se encuentra la asignatura Fundamentos del Desarrollo Sostenible. Aunque la cuestión de la movilidad forma parte de la línea 2 del Plan de Medio Ambiente de la URV, también se puede ligar con la línea 5, la ambientalización curricular (Alberich , Felipe, Aguilar y Saladié, 2012; Saladié y Jurado, 2013).

Se trata de una propuesta de prioridad alta y de fácil aplicación que implica la participación de la Facultad de Turismo y Geografía, de los responsables de los estudios que se imparten en la misma, de los profesores de las asignaturas de $2^{\circ}, 3^{\circ}$ y $4^{\circ}$ curso seleccionadas para realizar las sesiones informativas, de los responsables del Plan de Medio Ambiente de la URV y de la Cátedra Dow/URV de Desarrollo Sostenible. Esta última en tanto que encargada de desarrollar la línea 5 del Plan de Medio Ambiente.

\section{b) Sesión informativa con los estudiantes interesados en compartir coche.}

A pesar de que la URV dispone de un aplicativo web donde los miembros de la comunidad universitaria pueden ofrecer plazas en su coche como buscar ofertas y, más recientemente, ha desarrollado la herramienta virtual E-dit, que tiene por objetivo ayudar a encontrar trayectos comunes a través de una comunidad virtual, se estima oportuno que en las reuniones indicadas en la primera propuesta de este apartado se convoque a los estudiantes interesados en compartir coche a una nueva reunión. Su objetivo es facilitar la posibilidad de compartir coche como alternativa a la utilización individual del mismo, siempre y cuando el trayecto y los horarios lo hagan posible. Se propone realizar dos sesiones, una para aquellos estudiantes que mayoritariamente tienen horario de mañana y otra para aquellos que lo tienen de tarde. En la zona de aparcamiento del Campus Catalunya y del Campus Sescelades existen plazas reservadas para el estacionamiento de vehículos compartidos en el marco del proyecto E-dit.

$\mathrm{Al}$ igual que en la propuesta anterior, esta segunda también tienen una prioridad alta y es de fácil aplicación. En este caso los agentes implicados son la Facultad de Turismo y Geografía y los responsables del Plan de Medio Ambiente de la URV.

\section{c) Celebración del día europeo sin coches.}

El día 22 de septiembre se celebra el día europeo sin coches (free car day). En las reuniones informativas anteriormente mencionadas (propuestas a y b), se informará a los alumnos de este hecho y se animará a aquellos que se desplazan en transporte privado a que, en la medida que les sea posible, utilicen un medio de transporte alternativo más sostenible. La información sobre dichas alternativas les será facilitada en la reunión informativa que tendrá lugar a inicios del curso académico, es decir, unos 10 días antes del evento.

Se trata de una propuesta de prioridad alta y de fácil aplicación. El principal agente implicado es la Facultad de Turismo y Geografía, que puede establecer algún tipo de incentivo.

\section{d) Mayor compactación de los horarios de las asignaturas.}

En la medida en que sea posible, es necesario que haya una mayor compactación de los horarios de las asignaturas y que, para cada curso académico de cada uno de los dos grados que se imparten en la Facultad de Turismo y Geografía, se reduzcan las diferencias entre los diferentes días de la semana y entre los dos semestres.

La aplicación de esta medida, que consideramos de prioridad alta, presenta una cierta complicación debido a que implica a cuatro cursos de dos grados universitarios diferentes y que hay que tener en cuenta la disponibilidad y la adecuación de las aulas. En este caso el único agente implicado es la Facultad de Turismo y Geografía de la URV, especialmente los responsables de estudios del Grado en Turismo y del Grado en Geografía y Ordenación del Territorio. 


\section{e) Mayor frecuencia de autobuses entre Vila-seca y Reus.}

La conectividad entre Vila-seca y Reus mediante autobús es del todo insatisfactoria desde el punto de vista de la relación entre horarios de llegada/salida de los autobuses y horarios académicos, problema ya diagnosticado en plan para la mejora de la movilidad (URV, 2009). Casi un 18\% de los estudiantes de la muestra residen en Reus, pero de estos solamente un 2,2\% se desplazan en autobús y, en cambio, un $88,2 \%$ en coche. El hecho de no existir un autobús que llegue a Vila-seca antes de las 9 horas de la mañana, otro que llegue antes de las 15 horas de la tarde y otro que salga en dirección Reus poco después de las 19 horas de la tarde puede ser una causa que lo explique en buena medida. Por esta razón, desde la Facultad de Turismo y Geografía conjuntamente con el Ayuntamiento de Vila-seca y con la colaboración de la ATM, se tendría que solicitar a la empresa que tiene la concesión de la línea de autobuses entre Reus y Vila-seca un aumento en la frecuencia de autobuses que haga más factible la utilización de este medio de transporte por parte de los estudiantes residentes en Reus.

Esta propuesta se considera de prioridad alta pero con una aplicación difícil, puesto que el principal agente implicado es la compañía de autobuses.

\section{f) Establecimiento de una parada de autobús más cercana al Campus Vila-seca.}

Como en el caso anterior, esta propuesta también aparecía en el plan para la mejora de la movilidad (URV, 2009). Para llevarla a cabo es necesaria la modificación del recorrido de los autobuses con la incorporación de una nueva parada en la Vía Máxima, cerca de la calle Joanot Martorell donde se encuentra el Campus Vila-seca. Los agentes implicados serían los mismos que en la propuesta anterior.

Debido a que la distancia de la actual parada hasta la Facultad de Turismo y Geografía no es excesiva y la encuesta no muestra que sea una de las razones para la no utilización del transporte público, en este caso el autobús, consideramos esta propuesta como de prioridad media.

\section{CONCLUSIONES}

La movilidad de la comunidad universitaria de la URV es una de las líneas estratégicas del Plan de Medio Ambiente de la universidad pública de Tarragona y entre sus objetivos se encuentra alcanzar una movilidad más sostenible, aumentando el uso del transporte público colectivo y disminuyendo el uso del coche, especialmente si no es compartido. En este estudio se han analizado las pautas de movilidad de los estudiantes del Campus Vila-seca de la URV, una de las sedes universitarias que la URV dispone en la provincia de Tarragona, concretamente la situada en el municipio de Vila-seca, situado a menos de $10 \mathrm{~km}$ de la ciudad de Tarragona. La muestra está conformada por 191 estudiantes de los grados de Turismo y de Geografía y Ordenación del Territorio.

Del análisis se desprende que un $68,1 \%$ de los estudiantes residen en poblaciones situadas como máximo a $10 \mathrm{~km}$ de distancia del Campus, incluyendo Vila-seca. A pesar de ello, casi dos tercios de los encuestados se desplazan en coche desde su domicilio de residencia durante el curso académico y de éstos la mayoría van solos. La suma de los estudiantes que viajan en tren o en autobús sólo representa poco más de una cuarta parte de la muestra. Existen diferencias significativas teniendo en cuenta el municipio de residencia de los estudiantes. La mayoría de los residentes en Vila-seca hacen el recorrido a pie, aunque sólo son el 54,2\% y más de un tercio se desplazan en coche. Entre los residentes en Tarragona predomina el uso del autobús $(52,6 \%)$ seguido del coche $(40,4 \%)$. Tanto entre los estudiantes que residen en Reus como en Salou y Cambrils predomina el uso del coche, superando el 85\% del total. Finalmente, entre los que residen en el resto de localidades un 78,3\% también se desplazan en coche.

Los estudiantes encuestados han sido preguntados sobre la principal razón de utilizar un determinado medio de transporte. Un $41,5 \%$ de los que utilizan el coche manifestaron que el principal motivo era la rapidez del desplazamiento. Por su parte, un 26\% indicaron que la razón principal era la poca oferta de transporte público entre su localidad de residencia y el Campus Vila-seca. Entre los usuarios de autobús y de tren la razón principal en el uso de estos dos medios de transporte público colectivo era el precio con un $32,1 \%$, seguida muy de cerca por el hecho de no disponer de vehículo propio $(28,3 \%)$ y no disponer de licencia para conducir $(18,9 \%)$. Unas respuestas que dan a entender que cambiarían a coche en el caso que dispusieran de este tipo de vehículo. 
Los usuarios del vehículo privado han sido preguntados sobre si cambiarían a un transporte público colectivo en el caso que las razones aducidas para no utilizarlo no existiesen. La suma de la fracción individual de la respuesta de cada estudiante que se desplaza en coche da como resultado que un $62,2 \%$ haría el cambio.

Las universidades, tanto en el ámbito de la docencia como de la investigación y la gobernanza, juegan un papel clave en el fomento del desarrollo sostenible. En este sentido se han definido una serie de propuestas que permitan, con su aplicación, modificar y mejorar la realidad. Los estudiantes deben ser informados sobre los beneficios de utilizar el transporte público colectivo, en el caso que tengan esta opción, y también sobre el hecho que existe la posibilidad de compartir coche. También se propone celebrar el día europeo sin coches. Para aumentar la utilización del autobús se propone aumentar la frecuencia de autobuses, especialmente entre Reus y Vila-seca, así como establecer una parada más cercana al campus universitario de la URV.

Los resultados mostrados en este estudio nos dan una imagen de la situación en un momento determinado. En este sentido es necesario realizar el mismo análisis de forma sistemática con una periodicidad anual que permitirá vislumbrar la evolución en la movilidad de los estudiantes desde su lugar de residencia durante el curso académico hasta la Facultad de Turismo y Geografía de la URV, así como el impacto que hayan podido tener las propuestas presentadas en este artículo, en el caso que sean implementadas. No obstante, los nuevos análisis han de incluir tanto a los estudiantes como al Personal Docente e Investigador (PDI) y al Personal de Administración y Servicios (PAS).

\section{AGRADECIMIENTOS}

Este estudio ha sido realizado en el marco de las actividades de la Cátedra Dow/URV de Desarrollo Sostenible y ha contado con el apoyo de los proyectos GLOBALTUR (CS02011-23004/GEOG) y MOVETUR (CSO2014-51785-R), financiados por el Ministerio de Economía y Competitividad del Gobierno de España. El primer autor agradece el apoyo del Programa Serra Húnter de la Generalitat de Cataluña.

\section{REFERENCIAS}

Alberich, J., Felipe, B., Aguilar, E. y Saladié, O. (2012). La docència i la recerca en sostenibilitat i medi ambient a la Universitat Rovira i Virgili, Publicacions URV, Tarragona.

Alonso, B. (2002). Un análisis de movilidad de los alumnos de la Facultad de Ciencias Económicas y Empresariales de la Universidad de La Laguna, Documentos de Trabajo 2002-2005, Universidad de La Laguna, La Laguna.

Atherton, A. y Giurco, D. (2011). Campus sustainability: climate change, transport and paper reduction. International Journal of Sustainability in Higher Education, 12, 269-279.

Anable, J. (2005). Complacent car addicts or aspiring environmentalists? Identifying travel behavior segments using attitude theory. Transport Policy, 12, 65-78.

Balsas, C.J.L. (2003). Sustainable transportation planning on college campuses. Transport Policy, 10, 35-49.

Banister, D. (2008). The sustainable mobility paradigm. Transport Policy, 15, 73-80.

Bilbao, J. y Fernández, A. (2004). The influence of quality and price on the demand for urban transport: the case of university students. Transport Research Part A, 38, 607-614.

Bond, A. y Steiner, R.L. (2006). Sustainable campus transportation through transit partnership and transportation demand management: a case study from the Univeristy of Florida. Berkeley Planning Journal, 19, 125-142.

Brög, W., Erl, E. y Mense, N. (2004). Individualised marketing: changing travel behaviour for a better environment. Communicating environmentally sustainable transport: the role of soft measures, OECD, Paris, 83-97.

Button, K. y Nijkamp, P. (1997). Social change and sustainable transport. Journal of Transport Geography, 5, 215-218.

Camagni, R., Gibelli, M.C. y Rigamonti, P. (2002). Urban mobility and urban form: the social and environmental costs of different patterns of urban expansion. Ecological Economics, 40, 199-216.

Catalán, B., Saurí, D. y Serra, P. (2008). Urban sprawl in the Mediterranean? Patterns of growth and change in the Barcelona Metropolitan Region 1993-2000. Landscape and Urban Planning, 85, 174-184. 
Cebollada, A. y Miralles-Guasch, C. (2010). La movilidad en la Región Metropoltina de Barcelona: entre los nuevos retos y las viejas prácticas. Finisterra, XLV, 90, 33-47.

Chapman, L. (2007). Transport and climate change: a review. Journal of Transport Geography, 15, 354-367.

de las Rivas, J.L., Iglesias, F. y Lalana, J.L. (2011). Campus universitario de Valladolid. Integración urbana y movilidad. Bitácora, 18, 139-156.

Delmelle, E.M. y Delmelle, E.C. (2012). Exploring spatio-temporal commuting patterns in a university environment. Trasnport Policy, 21, 1-9

Efthymiou, D., Antoniou, C. y Waddell, P. (2013). Factors affecting the adoption of vehicle sharing systems by young drivers. Transport Policy, 29, 64-73.

Emanuel, R. y Adams, J.N. (2011). College students' perceptions of campus sustainability. International Journal of Sustainability in Higher Education, 12, 79-92.

ERF (2010). Pla de Medi Ambient de la Universitat Rovira i Virgili, Universitat Rovira i Virgili, Tarragona.

ERF (2012). Pla d'acció 2011-2015, Universitat Rovira i Virgili.

Evangelinos, K.I., Jones, N. y Panoriou, E.M. (2009). Challenges and opportunities for sustainability in regional universities: a case study in Mytilene, Greece. Journal of Cleaner Production, 17, 1154-1161.

Eriksson, L., Garvill, J. y Nordlund, A.M. (2008). Interrupting habitual car use: the importance of car habit strength and moral motivation for personal car use reduction. Transportation Research Part F, 11, 10-23.

Ferrer-Balas, D. (2004). Global environmental planning at the Technical University of Catalonia. International Journal of Sustainability in Higher Education, 5, 48-62.

Ferrer-Balas, D., Bruno, J., de Mingo, M. y Sans, R. (2004). Advances in education transformation towards sustainable development at the Technical University of Catalonia, Barcelona. International Journal of Sustainability in Higher Education, 5, 251-266.

García-Palomares, J.C. (2010). Urban sprawl and travel to work: the case of the metropolitan area of Madrid. Journal of Transport Geography, 18 (2), 197-213.

Green, D. y Wegener, M. (1997). Sustainable transport. Journal of Transport Geography, 5 (3), 177-190.

Hancock, L. y Nuttman, S. (2014). Engaging higher education institutions in the challenge of sustainability: sustainable transport as a catalyst for action. Journal of Cleaner Production, 62, 62-71.

Haque, M.M., Chin, H.C. y Debnath, A.K. (2013). Sustainable, safe, smart - three key elements of Singapore's evolving trasnport policies. Transport Policy, 27, 20-31.

Hensher, D.A. (1998). The imbalance between car and public transport use in urban Australia: why does it exist?. Transport Policy, 5, 193-204.

Hickman, R., Hall, P. y Banister, D. (2013). Planning more for sustainable mobility. Journal of Transport Geography, 33, 210-219.

Ibeas, A., dell'Olio, L. y Barreda, R. (2011). Citizen involvement in promoting sustainable mobility. Journal of Transport Geography, 19, 475-487.

Kerr, A., Lennon, A. y Watson, B. (2010). The call of the road: factors predicting students' car travelling intentions and behavior. Transportation, 37, 1-13.

Köhler, J., Whitmarsh, L., Nykvist, B., Shilperoord, M., Bergman, N. y Haxeltine, A. (2009). A transitions model for sustainable mobility. Ecological Economics, 12, 2985-2995.

Lambrechts, W., Mulà, I., Ceulemans, K., Molderez, I. y Gaeremynck, V. (2013). The integration of competences for sustainable development in higher education: an analysis of bachelor programs in management. Journal of Cleaner Production, 48, 65-73.

Lozano, R., Lukman, R., Lozano, FJ., Huisingh, D. y Lambrechts, W. (2013). Declarations for sustainability in higher education: becoming better leaders, through addressing the university system. Journal of Cleaner Production, 48, 10-19.

Lukman, R., Tiwary, A. y Azapagic, A. (2009). Towards greening a university campus: the case of the University of Maribor, Slovenia. Resources, Conservation and Recycling, 53, 639-644.

Mackett, R.L. (2001). Policies to attract drivers out of their cars for short trips. Transport Policy, 8, 295-306.

Mackett, R.L. (2003). Why do people use their cars for short trips?. Transportation, 30, 329-349. 
Mcrit (2013). Estudi de mobilitat al Campus de la URV a les Terres de l'Ebre, Universitat Rovira i Virgili, Tortosa, $80 \mathrm{pp}$.

Miralles-Guasch, C. (2010). Estrategias de participación ciudadana en la gestión de la movilidad y el transporte. La Universidad Autónoma de Barcelona como ejemplo. Scripta Nova, 14.

Miralles-Guasch, C. (2012). Las encuestas de movilidad y los referentes ambientales de los transportes. Eure, 38, 33-45.

Miralles-Guasch, C. y Cebollada, A. (2009). Movilidad cotidiana y sostenibilidad, una interpretación desde la geografía humana. Boletín de la AGE, 50, 193-216.

Miralles-Guasch, C. y Domen, E. (2010). Sustainable transport challenges in a suburban university: the case of the Autonomous University of Barcelona. Transport Policy, 17, 454-463.

Miralles-Guasch, C., Martínez, M. y Marquet, O. (2012). El uso del transporte privado. Percepciones individuales y contradicciones colectivas en un marco de sostenibilidad ambiental y social. Arquitectura, Ciudad y Entorno, 19, 95-110.

Moriarty, P. y Honnery, D. (2008). The prospects for global green car mobility. Journal of Cleaner Production, 16, 1717-1726.

Nejati, M. y Nejati, M. (2013). Assessment of sustainable university factors from the perspective of university students. Journal of Cleaner Production, 48, 101-107.

Pitsiava-Latinopoulou, M., Basbas, S. y Gavanas, N. (2013). Implementation of alternative transport networks in university campuses. The case of the Aristotle University of Thessaloniki, Greece. International Journal of Sustainability in Higher Education, 14, 310-323.

Pooley, C.G., Horton, D., Sscheldeman, G., Mullen, C., Jones, T., Tight, M., Jopson, A. y Chisholm, A. (2013). Policies for promoting walking and cycling in England: a view from the street. Transport Policy, 27, 66-75.

Prillwitz, J. y Barr, S. (2011). Moving towards sustainability? Mobility styles, attitudes and individual travel behavior. Journal of Transport Geography, 19, 1590-1600.

Pucher, J. y Dikjstra, L. (2003). Promoting safe walking an cycling to improve public health: lessons from the Netherlands and Germany. American Journal of Public Health, 93, 1509-1516.

Richardson, B.C. (2005). Sustainable transport: analysis frameworks. Journal of Transport Geography, 13, 29-39.

Roth, A. y Kaberger, T. (2002). Making transport systems sustainable. Journal of Cleaner Productions, 10, 361-371.

Saladié, O., Alberich, J., Felipe, B. y Aguilar, E. (2013). La diagnosis como primera fase del proceso de ambientalización curricular en la Universitat Rovira i Virgili. I Encuentro Universitario de Sostenibilización Curricular Universidad Europea, Villaviciosa de Odón (Madrid).

Saladié, O. y Jurado, J. (2013). Les competències de caràcter ambiental als estudis de grau de la URV. Càtedra Dow/URV de Desenvolupament Sostenible. Universitat Rovira i Virgili, Tarragona (informe interno).

Seguí-PonS, J.M., Ruiz, M. y Luna, M. (2013). Movilidad y transportes en el acceso al Campus de la Universitat de les Illes Balears: una perspectiva de género. XIII Congreso de Geógrafos Españoles. Espacios Insulares y de frontera, una visión geográfica, Asociación de Geógrafos Españoles, Palma de Mallorca, 685-695.

Sedlacek, S. (2013). The role of universities in fostering sustainable development at the regional level. Journal of Cleaner Production, 48, 74-84.

Shannon, T., Giles-Corti, B., Pikora, T., Bulsara, M., Shilton, T. y Bull, F. (2006). Active commuting in a university setting: assessing commuting habits and potential for modal change. Transport Policy, 13, 240-253.

Steg, L. y Gifford, R. (2005). Sustainable transportation and quality of life. Journal of Transport Geography, 13, 59-69.

Tolley, R. (1996). Green campuses: cutting the environmental cost of commuting. Journal of Transport Geography, 4, 213-217. 
URV (2009). Pla de Millores de la mobilitat als centres universitaris de la Rovira i Virgili al Camp de Tarragona. Propostes. Pla d'acció 2011-2015, Universitat Rovira i Virgili, Tarragona.

URV (2013). Evolució d'emissions de GEH 2012, Documents 2013, Universitat Rovira i Virgili, Tarragona.

Velazquez, L., Munguia, N., Platt, A. y TADDEI, J. (2006). Sustainable univeristy: what can be the matter? Journal of Cleaner Production, 14, 810-819.

Vergragt, P.J. y Szejnwald, H. (2007). Sustainable mobility: from technological innovation to social learning. Journal of Cleaner Production, 15, 1104-1115.

Villanueva, K., Giles-Corti, B. y McCormack, G. (2008). Achieving 10,000 steps: a comparison of public transport users and drivers in a University setting. Preventive Medicine, 47, 338-341.

Whalen, K.E., Páez, A. y Carrasco, J.A. (2013). Mode choice of university students commuting to school and the role of active travel. Journal of Transport Geography, 31, 132-142.

Woodcock, J., Banister, D., Edwards, P., Prentice, A.M. y Robert, I. (2007). Energy and transport. Lancet, 370, 1078-1088. 